\title{
Sonic Hedgehog/Patch Pathway Activated
}

National Cancer Institute

\section{Source}

National Cancer Institute. Sonic Hedgehog/Patch Pathway Activated. NCI Thesaurus.

Code C82326.

A genetic finding indicating that the sonic hedgehog/patch pathway is activated. 